\title{
Editorial
}

\section{Networked Systems with Incomplete Information}

\author{
Zidong Wang, ${ }^{1}$ Bo Shen, ${ }^{2}$ Hongli Dong, ${ }^{3,4}$ Xiao $\mathrm{He}^{5}$ and Jun $\mathrm{Hu}^{6}$ \\ ${ }^{1}$ Department of Computer Science, Brunel University London, Uxbridge, Middlesex UB8 3PH, UK \\ ${ }^{2}$ School of Information Science and Technology, Donghua University, Shanghai 200051, China \\ ${ }^{3}$ College of Electrical and Information Engineering, Northeast Petroleum University, Daqing 163318, China \\ ${ }^{4}$ Research Institute of Intelligent Control and Systems, Harbin Institute of Technology, Harbin 150001, China \\ ${ }^{5}$ Department of Automation, Tsinghua University, Beijing 100084, China \\ ${ }^{6}$ Department of Applied Mathematics, Harbin University of Science and Technology, Harbin 150080, China \\ Correspondence should be addressed to Zidong Wang; zidong.wang@brunel.ac.uk
}

Received 25 December 2014; Accepted 25 December 2014

Copyright (C) 2015 Zidong Wang et al. This is an open access article distributed under the Creative Commons Attribution License, which permits unrestricted use, distribution, and reproduction in any medium, provided the original work is properly cited.

In this special issue, we have solicited submissions from electrical engineers, control engineers, computer scientists, and mathematicians. After a rigorous peer review process, 18 papers have been selected that provide overviews, solutions, or early promises, to manage, analyse, and interpret dynamical behaviours of networked systems. These papers have covered both the theoretical and practical aspects of networked system with incomplete information in the broad areas of dynamical systems, mathematics, statistics, operational research, and engineering.

In this special issue, there is a survey paper on the recent advances of control and filtering problems for TakagiSugeno (T-S) fuzzy systems with network-induced phenomena. Specifically, in the paper entitled "Analysis, Filtering, and Control for Takagi-Sugeno Fuzzy Models in Networked Systems" by S. Zhang et al., the focus is to provide a timely review on some recent advances on the T-S fuzzy control and filtering problems with various network-induced phenomena. Because of the advantages in dealing with various nonlinear systems, the fuzzy logic theory has great success in industry applications. Among various kinds of models for fuzzy systems, the T-S fuzzy model is quite popular due to its convenient, simple dynamic structure and the capability of approximating any smooth nonlinear function to any specified accuracy within any compact set. This survey discusses a variety of T-S fuzzy control and filtering issues with network-induced phenomena in great detail firstly. Four network-induced phenomena (communication delays, packet dropouts, signal quantization, and randomly occurring uncertainties (ROUs)) are introduced. Both theories and techniques for dealing with the controller or filter design are systematically reviewed. Then, some latest results on T$S$ fuzzy control/filtering problems (bilinear T-S fuzzy model, event-based fuzzy control, fuzzy filtering with multiple network-induced phenomena, $l_{2}-l_{\infty}$ fuzzy filtering, $H_{-} / H_{\infty}$ fault detection, $H_{\infty}$ filtering with unknown membership functions, and nonfragile $H_{\infty}$ fuzzy filtering) for networked systems are surveyed and some challenging issues for future research are raised. Finally, some conclusions are drawn and several possible related research directions are pointed out.

In the past decades, the stability analysis of the networked systems has attracted much research attention. In the work entitled "Uniform Stability Analysis of Fractional-Order BAM Neural Networks with Delays in the Leakage Terms" by X. Yang et al., the uniform stability analysis is studied for a class of fractional-order BAM neural networks with delays in the leakage terms. By introducing a novel norm, several delay-dependent sufficient conditions are obtained to ensure the uniform stability of the proposed system by using inequality technique and analysis method. Moreover, sufficient conditions are established to guarantee the existence, uniqueness, and uniform stability of the equilibrium point. Three simulation examples are given to demonstrate the effectiveness of the obtained results. It should be pointed out that it is possible to extend the main results of this paper to other complex systems and establish novel stability conditions 
with less conservatism by using more up-to-date techniques. The $\mu$-stability issue is discussed in "Global $\mu$-Stability of Impulsive Complex-Valued Neural Networks with Leakage Delay and Mixed Delays" by X. Chen et al. for complexvalued neural networks (CVNNs) with leakage delay, discrete delay, and distributed delay under impulsive perturbations. The $\mu$-stability is the concept for the purpose of unifying the exponential stability, power-rate stability, and log-stability of neural networks. CVNN is an extension of real-valued neural network which has been applied in physical systems dealing with electromagnetic, light, ultrasonic, and quantum waves. Based on the homeomorphism mapping principle of complex domain, a sufficient condition for the existence and uniqueness of the equilibrium point of the addressed CVNNs is proposed in terms of linear matrix inequality (LMI). By constructing appropriate Lyapunov-Krasovskii functionals and employing the free weighting matrix method, several delay-dependent criteria for checking the global $\mu$-stability of the CVNNs are established in LMIs. As direct applications of these results, several criteria on the exponential stability, power-stability, and log-stability are obtained. In the paper entitled "A Switched Approach to Robust Stabilization of Multiple Coupled Networked Control Systems" by M. Yu et al., multiple coupled networked controlled systems (NCSs) with norm-bounded parameter uncertainties and multiple transmissions are considered. All the nodes in the proposed systems act over a limited bandwidth communication channel. The state information of every subsystem is split into different packets and there is only one packet of the subsystem that can be transmitted at a time. Based on the toking bus protocol, the nodes are arranged logically into a ring and transmit the corresponding packets in a prefixed circular order. Then, the proposed multiple NCSs can be modelled as periodic switched systems. Furthermore, the robust stabilization issue is dealt with by applying the switched system theory. State feedback controllers are constructed in terms of LMIs. A numerical example is given to show that the coupled NCSs considered can be effectively stabilized via the designed controller.

Control and fault estimation problems for stochastic systems have been of interest of many researchers during the past decades. In the paper entitled "Robust $H_{\infty}$ Control for a Class of Discrete Time-Delay Stochastic Systems with Randomly Occurring Nonlinearities" by Y. Wang et al., the robust $H_{\infty}$ problem is studied for a class of discrete time-delay stochastic systems with randomly occurring nonlinearities (RONs). It is assumed that all the system matrices contain the parameter uncertainties. The stochastic disturbances are both state- and control-dependent, and the RONs satisfy the sector boundedness conditions. The purpose of the problem proposed is to design a state feedback controller such that, for all admissible uncertainties, nonlinearities, and timedelays, the closed-loop system is robustly asymptotically stable in the mean square, and a prescribed $H_{\infty}$ disturbance rejection attenuation level is also guaranteed. By using the Lyapunov stability theory and stochastic analysis tools, a LMI approach is developed to derive sufficient conditions ensuring the existence of the desired controllers, where the conditions are dependent on the lower and upper bounds of the time-varying delays. The explicit parameterization of the desired controller gains is also given. The problem of $H_{\infty}$ control for network-based 2D systems with missing measurements is investigated in " $H_{\infty}$ Control for NetworkBased 2D Systems with Missing Measurements" by X. Bu et al. A state feedback controller is designed such that the closed-loop 2D stochastic system is mean-square asymptotic stability and has $H_{\infty}$ disturbance attenuation performance. A sufficient condition is derived in terms of LMIs technique, and formulas can be given for the control law design. The result is also extended to more general cases where the system matrices contain uncertain parameters. Numerical examples are also provided to show the effectiveness of proposed approach. In the work entitled "Krein Space-Based $H_{\infty}$ Fault Estimation for Discrete Time-Delay Systems" by $\mathrm{X}$. Song and $\mathrm{X}$. Yan, the finite-time $H_{\infty}$ fault estimation issue is investigated for linear time-delay systems where the delay appears in both state output and measurement output. Firstly, the design of finite horizon $H_{\infty}$ fault estimation is converted into a minimum problem of certain quadratic form. Then, a sufficient and necessary condition for the existence of the desired $H_{\infty}$ fault estimator is derived by employing the Krein-space theory. A solution of the desired $H_{\infty}$ fault estimator is obtained by recursively computing a partial difference Riccati equation which has the same dimension as the original systems. Therefore, solving a high dimension Riccati equation is avoided compared with the conventional augmented method. A numerical example is given to demonstrate the effectiveness of the approach.

Circulant type matrices have significant applications in network systems. In the work entitled "Equalities and Inequalities for Norms of Block Imaginary Circulant Operator Matrices" by X. Jiang and K. Hong, the block imaginary circulant operator matrices are studied. Firstly, by combining the special properties of block imaginary circulant operator matrix with unitarily invariant norm, several norm equalities are obtained. It should be pointed out that the norm in consideration is the weakly unitarily invariant norm. The usual operator norm and Schatten $p$-norm are included. Then, several pinching type inequalities are presented by the triangle inequality and the invariance property of unitarily invariant norms. Furthermore, some special cases and examples are considered. Circulant and left circulant matrices with Fermat and Mersenne numbers are considered in "Exact Inverse Matrices of Fermat and Mersenne Circulant Matrix" by Y. Zheng and S. Shon. Moreover, the exact determinants and the inverse matrices of Fermat and Mersenne left circulant matrix are given. The nonsingularity of these special matrices is discussed. In the paper entitled "Norms and Spread of the Fibonacci and Lucas RSFMLR Circulant Matrices" by $\mathrm{W}$. Xu and Z. Jiang the norms and spread of Fibonacci row skew first-minus-last right (RSFMLR) circulant matrices are investigated as well as the Lucas RSFMLR circulant matrices. Firstly, these two kinds of special matrices are defined. Then, the lower and upper bounds for the spectral norms of these matrices are proposed as well as the upper bounds for the spread of these matrices. Afterwards, some corollaries related to norms of Hadamard and Kronecker products of these matrices are obtained, respectively. The determinants and 
inverses of Tribonacci circulant type matrices are discussed in "Explicit Form of the Inverse Matrices of Tribonacci Circulant Type Matrices" by L. Liu and Z. Jian. The definition of Tribonacci circulant type matrices is given firstly. Then, the invertibility of Tribonacci circulant type matrices is studied. Based on constructing the transformation matrices, both the determinant and the inverse matrix are derived. Furthermore, by utilizing the relation between left circulant, $g$-circulant matrices, and circulant matrix, the invertibility of Tribonacci left circulant and Tribonacci $g$-circulant matrices is also studied. Finally, the determinants and inverse matrices of these matrices are presented, respectively. A future research direction is pointed out at last. In the paper entitled "Analysis of the Structured Perturbation for the BCSCB Linear System" by $\mathrm{X}$. Tang and $\mathrm{Z}$. Jian, the analysis problem associated with the BCSCB matrix is considered. The BCSCB matrix is an extension of the circulant matrix and skew circulant matrix. Firstly, the form of the BCSCB matrix is obtained based on the style spectral decomposition of the basic circulant matrix and the basic skew circulant matrix. Then, the structured perturbation analysis for BCSCB linear system is proposed, which includes the condition number and relative error of the BSCSB linear system. A new approach is presented to derive the minimal value of the perturbation bound, which is only related to the perturbation of the coefficient matrix and the vector. Simultaneously, the algorithm for the optimal backward perturbation bound is developed.

As is well known, the analysis of issues on network systems has important significance. In the paper entitled "On the Incidence Energy of Some Toroidal Lattices" by J.-B. Liu, et al., the closed-form formulae expressing the incidence energy of the 3.12.12 lattice and triangular kagomé lattice are derived as well as $S(m, n)$ lattice. The calculations of the energy of graphs become a popular topic of research. However, it is not an easy task to deal with the problem of the asymptotic incidence energy of various lattices with the free boundary. By utilizing the applications of analysis approach with the help of software calculation, the explicit asymptotic values of the incidence energy in these lattices are derived simultaneously. This developed method can be used widely to handle the asymptotic behaviour of other lattices and can obtain some useful results simultaneously. For the purpose of studying the distribution of evolving networks, a kind of evolving network is proposed in "Asymptotic Degree Distribution of a Kind of Asymmetric Evolving Network" by $\mathrm{Z}$. Li et al., where the model is a combination of preferential attachment model and uniform model. The distribution of the number of vertices with given degree is studied as well as the asymptotic degree distribution. It is shown that the proportional degree sequence obeys power law, exponential distribution, and other forms according to the relation of the degree and parameter $m$. In the work entitled "Partial Synchronizability Characterized by Principal QuasiSubmatrices Corresponding to Clusters" by G. Zhang et al., a partial synchronization problem is studied in an oscillator network. In order to investigate the partial synchronization, the concept on a principal quasi-submatrix corresponding to the topology of a cluster is proposed. A novel criterion on partial synchronization is developed based on the analysis of principal quasi-submatrices corresponding to the clusters. The proposed criterion is not distinctly dependent on the intercluster couplings or the topology matrix of the whole network. If a network is composed of a large number of nodes, the enormous amount of calculation can be reduced by replacing the coupling matrix with several quasi-submatrices. Therefore, this criterion provides a novel index of partial synchronizability. It is shown that different types of partial synchronization occur in a star-global network when the coupling strength is increased. The proposed approach for partial synchronization might be applicable to the complex networks with networked induced phenomena. A backbone extraction heuristic with incomplete information (BEHwII) is investigated in "Extracting Backbones from Weighted Complex Networks with Incomplete Information" by L. Qian et al. The presence of the backbone is the signature or the abstraction of the nature of complex systems and can provide huge help for understanding them in more simplified forms. For the purpose of extracting backbones from largescale weighted networks, a novel filter-based approach is presented which only needs incomplete information and then invokes the iteratively local search scheme for improving the efficiency. First, a strict filtering rule is designed to determine edges to be preserved or discarded. Then, a local search model is proposed to examine part of edges in an iterative way. Experimental results on four real-life networks demonstrate the advantage of BEHwII over the classic disparity filter method by either effectiveness or efficiency validity.

Recently, the application of networked systems has attracted a great deal of research interest. The data communication networks play an important role during the development of smart grid. Since the data communication network in smart gird is affected by plenty of decisive factors, different decision-making problems are presented according to the variable factors. In the paper entitled "Real-Time Pricing Decision Making for Retailer-Wholesaler in Smart Grid Based on Game Theory" by Y. Dai and Y. Gao, a novel game-theoretical decision-making scheme is investigated for electricity retailers and wholesaler in the smart grid with demand side management (DSM). The interaction between two retailers and their wholesaler is modelled by a two-stage dynamic game where the competition between two retailers is considered. According to the different action order between retailers and their wholesaler, two different game models are developed. The subgame perfect Nash equilibrium (SPE) for this game is determined through backward induction. It is shown that the wholesaler wants to decentralize certain management powers to the retailers through analysing the equilibrium revenues of the retailers for different situations. Imposing legal restrictions on the wholesaler's discretionary policy suggests that the time-inconsistency problem is mitigated. The packing problem of unit equilateral triangles is investigated in "A New Quasi-Human Algorithm for Solving the Packing Problem of Unit Equilateral Triangles" by R. Wang et al. The packing problem of unit equilateral triangles offers broad prospects in different fields including the network resource optimization. This problem is nondeterministic polynomial (NP) hard and has the feature of continuity. A novel quasi-human algorithm for solving this 
problem is proposed according to the characteristic of the unit equilateral triangles and in the base of analysis of the general triangles packing problems. Time complexity analysis and the calculation results indicate that the proposed method is a polynomial time algorithm, which provides the possibility to solve the packing problem of arbitrary triangles.

\section{Acknowledgments}

This special issue is a timely reflection of the research progress in the area of networked systems with incomplete information. We would like to acknowledge all authors for their efforts in submitting high-quality papers. We are also very grateful to the reviewers for their thorough and on-time reviews of the papers.

Zidong Wang

Bo Shen

Hongli Dong

Xiao He

Jun Hu 


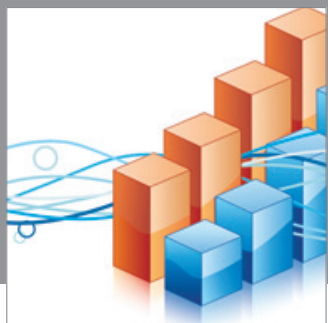

Advances in

Operations Research

mansans

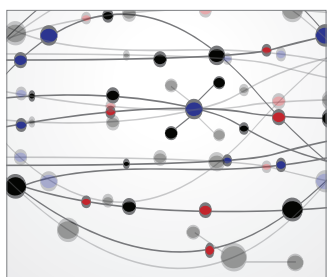

The Scientific World Journal
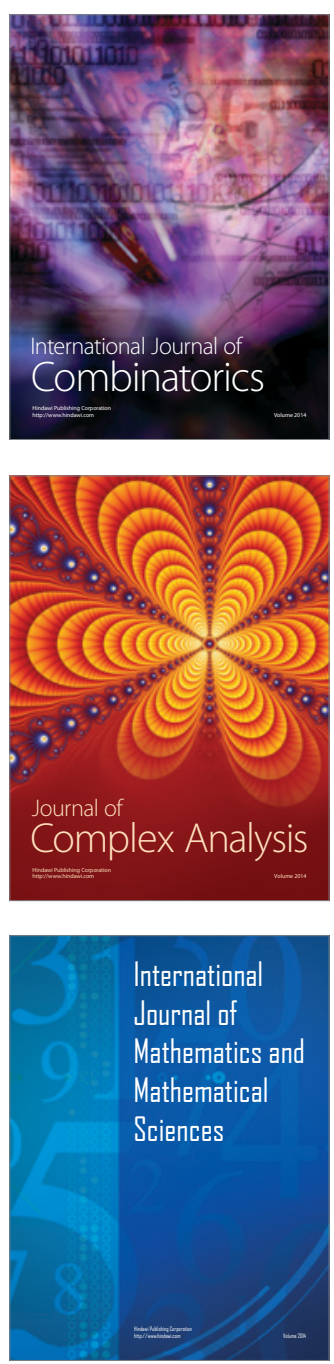
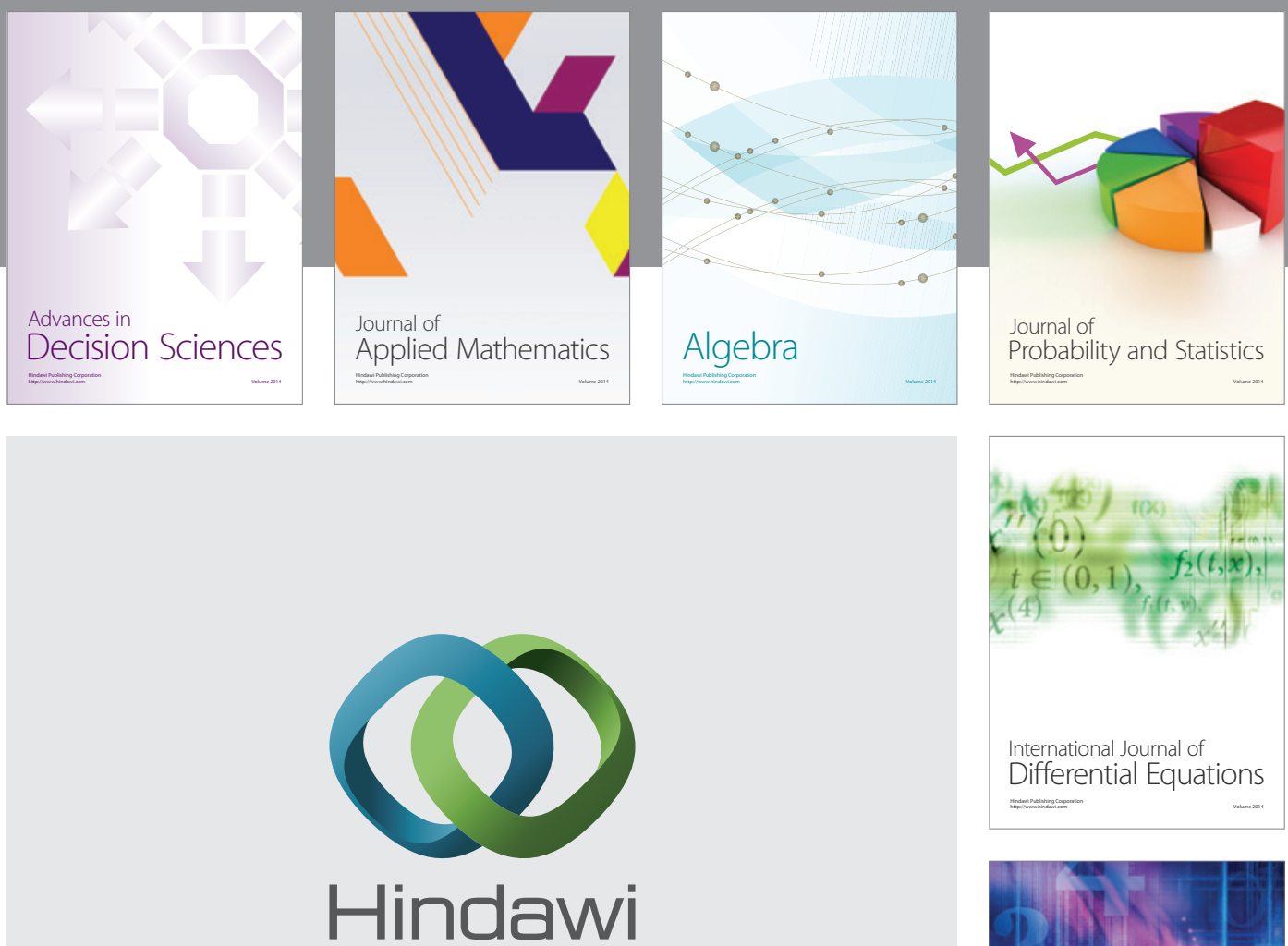

Submit your manuscripts at http://www.hindawi.com
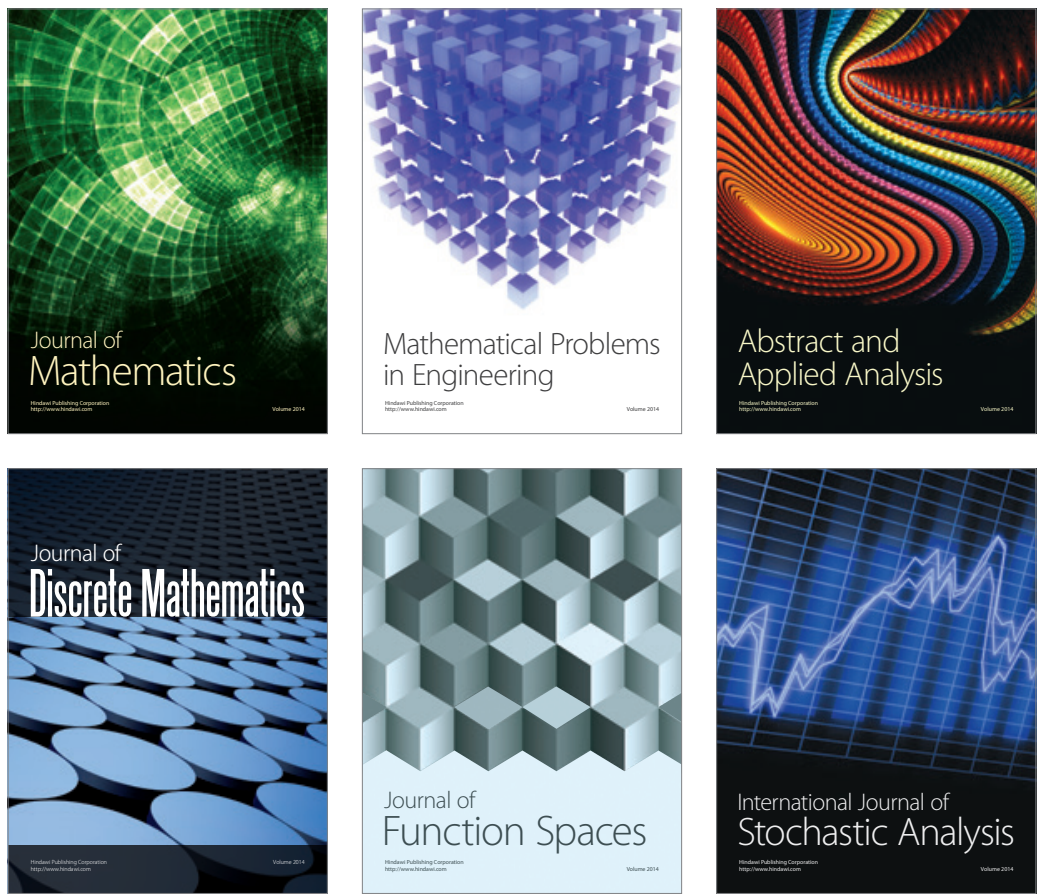

Journal of

Function Spaces

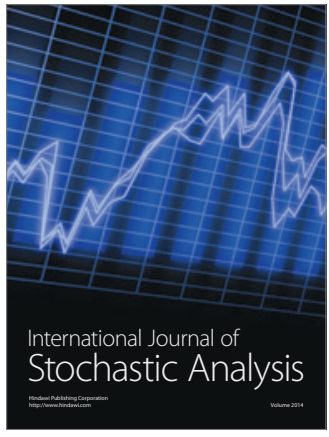

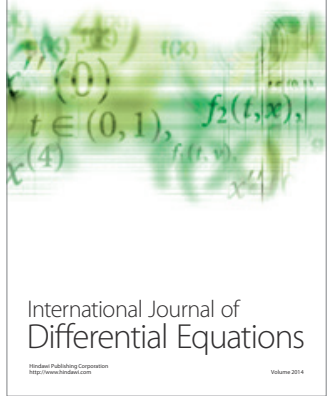
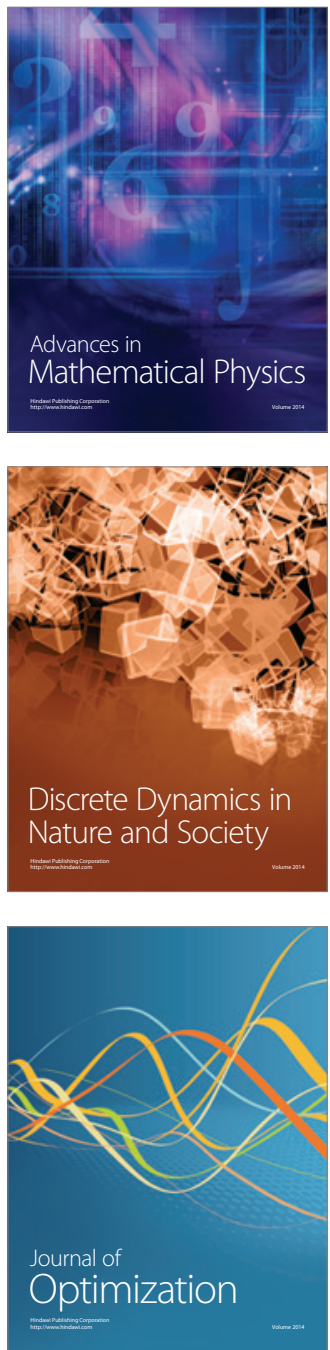